\section{Stephan Martin}

\section{Interventionsstudien zur \\ Lebensstilintervention}

In epidemiologischen Studien konnte gezeigt werden, dass Lebensstilfaktoren mit einer Vielzahl an Erkrankungen assoziiert sind. Ganz im Vordergrund steht der Diabetes mellitus Typ 2 , der in den vergangenen Jahren weltweit dramatisch angestiegen ist.

In einer Vielzahl an randomisierten kontrollierten Studien konnte auch ein kausaler Zusammenhang von Diabetes und Lebensstilfaktoren nachgewiesen werden (Abb. 1). Bei der weltweit ersten Studie zur Prävention des Diabetes mellitus Typ 2, der Da Qing Diabetes-Präventionsstudie, wurden im Jahr 1986 in China insgesamt 577 Erwachsene eingeschlossen, die eine pathologische Glukosetoleranz (impaired glucose tolerance (IGT)) hatten [1]. Nach 6 Jahren konnte im Vergleich zur Kontrollgruppe das Diabetesrisiko je nach Intervention um 31-46\% reduziert werden. Auch in einer Nachanalyse nach 23 Jahren konnte gezeigt werden, dass der Effekt der Lebensstiländerung über die Interventionszeit erhalten geblieben ist [2]. Diese Arbeit hat aber auch im Rahmen einer kontrollierten Studie gezeigt, dass sich durch eine Änderung des Lebensstils die Lebenserwartung beeinflussen lässt. So lag die kumulative Inzidenz der kardiovaskulären Mortalität in den Interventionsgruppen bei $11,9 \%$, gegenüber $19,6 \%$ in der Kontrollgruppe, was einer $41 \%$ igen Risikoreduktion entspricht $(p=0,033)$. Auch die Gesamtmortalität war in der LebensstilInterventionsgruppe signifikant re-

\title{
Lebensstilintervention und Diabetes mellitus Typ 2
}

duziert. Diese Studie zeigt somit, dass eine nur 6 Jahre andauernde Lebensstilintervention bei Personen mit Prädiabetes nicht nur Langzeitzeiteffekte auf die Entwicklung eines Diabetes mellitus Typ 2, sondern auch auf die Mortalität hat. Weitere Präventionsstudien wurden in Finnland [3,4] und den USA [5, 6] durchgeführt. Auch hier kam es zu einer Reduktion der Diabetesentwicklung, deren Wirksamkeit auch viele Jahre nach Beendigung der Interventionsphase in Form einer reduzierten Diabetesrate nachgewiesen werden konnte.

Auch beim manifesten Diabetes mellitus Typ 2 können metabolische Parameter durch eine Lebensstiländerung signifikant verbessert werden. In der Look-AHEAD-Studie Studie, der bisher grössten Lebensstil-Interventionsstudie beim manifesten Diabetes, wurden 5145 übergewichtige oder adipöse Personen mit Diabetes mellitus Typ 2 eingeschlossen [7]. In der ein- jährigen Interventionsstudie kam es zu einer signifikanten Reduktion von HbAlc und Blutdruck, wobei zusätzlich die antidiabetische und antihypertensive Therapie reduziert wurde. Der primäre Endpunkt der Studie - ein kombinierter Endpunkt aus Tod aufgrund von kardiovaskulären Ereignissen, nicht tödlichen Myokardinfarkten und Apoplexen sowie Hospitalisierung aufgrund von Angina pectoris - konnte jedoch nicht erreicht werden. In einer Nachanalyse wurden die Ergebnisse der Look-AHEAD-Studie in Abhängigkeit des Erfolgs einer Lebensstiländerung auf die primären und sekundären Endpunkte analysiert, denn in jeder Gruppe gab es erfolgreiche und nicht erfolgreiche Personen [8]. Personen, die im ersten Jahr mindestens $10 \%$ des Körpergewichts verloren, hatten in einem medianen Follow-up von 10,2 Jahren ein 21\% niedrigeres Risiko für den primären Endpunkt ( $\mathrm{p}=0,034)$.

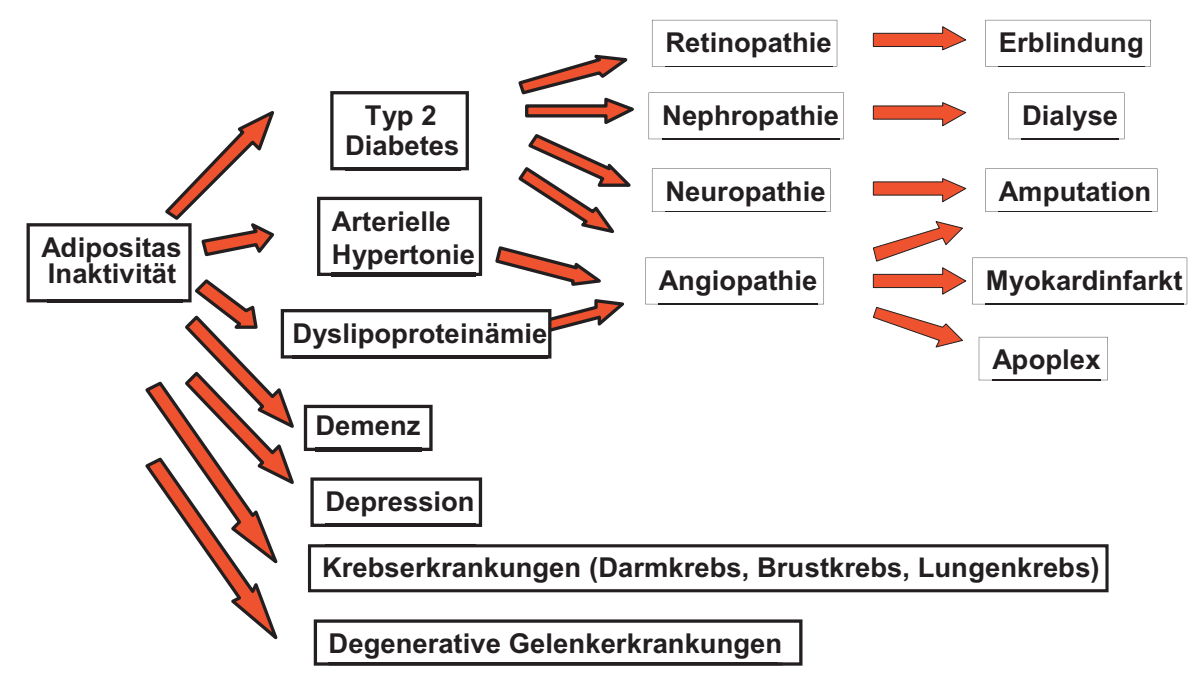

Abb. 1. Auswirkungen von Adipositas und Inaktivität.

\section{KARGER}

(c) 2017 S. Karger GmbH, Freiburg

Fax +497614520714
Prof. Dr. Stephan Martin

Westdeutsches Diabetes- und Gesundheitszentrum (WDGZ)

Verbund Katholischer Kliniken Düsseldorf (VKKD)

Hohensandweg 37, 40591 Düsseldorf, Deutschland

stephan.martin@vkkd-kliniken.de 
Es stellt sich jedoch die Frage, wie die Lebensstilintervention aussehen sollte. In allen zuvor zitierten Studien wurden die Patienten dazu angehalten, den Fettgehalt der Nahrung auf weniger als $30 \%$ zu reduzieren. Später gestartete Studien schlagen hier aber einen ganz anderen Weg ein. Bei der PREDIMED-Studie [9] wurden insgesamt 7447 Personen mit einem erhöhten kardiovaskulären Risiko im Alter zwischen 55 und 80 Jahren eingeschlossen. Während die Kontrollgruppe die übliche fettarme Kost empfohlen bekam, sollten die Teilnehmer der Interventionsgruppe eine mediterrane Ernährung zu sich nehmen, wobei eine Gruppe vermehrt Olivenöl zu sich nehmen sollte - sie bekam pro Woche 11 Olivenöl geschenkt -, während die zweite Interventionsgruppe täglich 30 g Nüsse (30 g/Tag) erhielt. Die Studie musste nach 4,8 Jahren abgebrochen werden, da der primäre Endpunkt bestehend aus Myokardinfarkt, Apoplex oder Tod aufgrund eines kardiovaskulären Ereignisses in der Gruppe mit der Olivenölsupplementierung um 30\% und in der mit Nüssen behandelten Gruppe um 28\% signifikant niedriger als in der Gruppe mit einer fettarmen Ernährung lag. In einer Subgruppe von Teilnehmern ohne Diabetes konnte der Einfluss dieser Ernährungsumstellung auf die Diabetesrate untersucht werden [10]. Dabei zeigte sich in der Olivenöl-Gruppe ein signifikant um $40 \%$ reduziertes Diabetesrisiko im Vergleich zur Kontrollgruppe. In der Nuss-Gruppe lag die Rate ebenfalls um $18 \%$ niedriger, war jedoch nicht statistisch signifikant. Auch beim neu diagnostizierten Diabetes mellitus Typ 2 scheint eine mediterrane/fettreiche Ernährung günstig zu sein. In der MÉDITA-Studie [11, 12] wurden Patienten mit neu diagnostiziertem Diabetes mellitus Typ 2 eingeschlossen, aber nicht sofort medikamentös behandelt. Die Ärzte, welche die Entscheidung über den Beginn einer medikamentösen Diabetestherapie fällten, waren «geblindet». Durch eine mediterrane/ fettreiche - im Vergleich zu einer fettarmen/kohlenhydratreichen - Kost konnte die Notwendigkeit einer medikamentösen Therapie um 2 Jahre verzögert werden.

Die Umsetzung dieser Evidenz, die eindeutig für eine breite Anwendung von Lebensstiländerungen zur Behandlung des Diabetes mellitus Typ 2 spricht, ist in der täglichen Praxis schwierig. Durch neue technische Möglichkeiten ergeben sich jedoch neue Therapieansätze, welche die Hausärzte bei der Behandlung von $\mathrm{Pa}-$ tienten mit Diabetes mellitus Typ 2 unterstützen. Am Westdeutschen Diabetes- und Gesundheitszentrum (WDGZ) haben wir das «Telemedizinische Lifestyle Programm» (TeLiPro) entwickelt und zusammen mit dem Deutschen Institut für Telemedizin und Gesundheitsförderung (DITG) im Rahmen einer randomisierten klinischen Studie evaluiert [13]. Das Programm sieht vor, dass Patienten einen Schrittzähler, ein Blutzuckermessgerät und eine Waage mit telemedizinischen Datenübertragungen zur Verfügung gestellt bekommen. Zusätzlich erhalten die Teilnehmer ein telemedizinisches Coaching einschliesslich eines mentalen medizinischen Motivationsprogramms sowie ein strukturiertes Formuladiätprogramm mit regelmässiger Blutzuckerselbstkontrolle über 12 Wochen. Im Vergleich zu einer Kontrollgruppe kam es in der TeLiPro-Gruppe während der Interventionsphase $\mathrm{zu}$ einer $\mathrm{HbAlc-Ab-}$ senkung um $-1,1 \%$ gegenüber $-0,2 \%$ in der Kontrollgruppe $(\mathrm{p}<0,001)$, die nur Schrittzähler und Waage erhielt. Auch die antidiabetische Medikation konnte in der TeLiPro-Gruppe signifikant reduziert und die Insulintherapie halbiert werden. Auch bei BodyMass-Index, Blutdruck, Lebensqualität und Essverhalten kam es zu einer signifikanten Verbesserung. Eine Nachbeobachtung der Patienten über 1 Jahr zeigte, dass die signifikanten Reduktionen der kardiometabolischen Risikofaktoren bestehen blieben. Das Besondere am TeLiPro ist, dass die Patienten durch das DITG telemedizinisch gecoacht wurden, aber gleichzeitig in der Betreuung durch den behandelnden Hausarzt blieben. Dieser hat die üblichen DMP(Disease-Management-Programm)-Untersuchungen durchgeführt und dem DITG-Studienzentrum die Laborwerte zur Verfügung gestellt.

Der in Grossbritannien sehr renommierte Mediziner Sir Muir Gray hat kürzlich den Diabetes mellitus Typ 2 als «walking deficiency syndrome» bezeichnet und davor gewarnt, dass Bezeichnungen wie Diabetes mellitus Typ 2 oder metabolisches Syndrom «makes you think it's like rheumatoid arthritis or a real disease». Die hier dargestellten Studien geben ihm sicher Recht, denn wir werden das Thema Diabetes mellitus Typ 2 sicher hinterfragen müssen. Sollte die Gesellschaft der Meinung sein, den Diabetes mellitus Typ 2 als ganz normale Erkrankung anzusehen, wird dies die Legitimation sein, den medikamentösen Weg mit 3-4 Diabetesmedikamenten weiter zu beschreiten. Sehen wir den Diabetes mellitus Typ 2 als «walking deficiency syndrome», müssen wir in die Änderung des Lebensstils investieren. 


\section{Literatur}

1 Pan XR, Li GW, Hu YH, Wang JX, Yang WY, An ZX, Hu ZX, Lin J, Xiao JZ, Cao HB, Liu $P A$, Jiang $X G$, Jiang YY, Wang JP, Zheng $H$, Zhang $\mathrm{H}$, Bennett PH, Howard BV: Effects of diet and exercise in preventing NIDDM in people with impaired glucose tolerance. The Da Qing IGT and Diabetes Study. Diabetes Care 1997;20:537-544.

2 Li G, Zhang P, Wang J, An Y, Gong Q, Gregg EW, Yang W, Zhang B, Shuai Y, Hong J, Engelgau MM, Li H, Roglic G, Hu Y, Bennett PH: Cardiovascular mortality, all-cause mortality, and diabetes incidence after lifestyle intervention for people with impaired glucose tolerance in the Da Qing Diabetes Prevention Study: a 23-year follow-up study. Lancet Diabetes Endocrinol 2014;2:474-480.

3 Tuomilehto J, Lindstrom J, Eriksson JG, Valle TT, Hamalainen H, Ilanne-Parikka P, Keinanen-Kiukaanniemi S, Laakso M, Louheranta A, Rastas M, Salminen V, Uusitupa M: Prevention of type 2 diabetes mellitus by changes in lifestyle among subjects with impaired glucose tolerance. N Engl J Med 2001;344:1343-1350.

4 Lindström J, Peltonen M, Eriksson JG, IlanneParikka P, Aunola S, Keinänen-Kiukaanniemi S, Uusitupa M, Tuomilehto J; Finnish Diabetes Prevention Study (DPS): Improved lifestyle and decreased diabetes risk over 13 years: long-term follow-up of the randomised Finnish Diabetes Prevention Study (DPS). Diabetologia 2013;56:284-293.
5 Knowler WC, Barrett-Connor E, Fowler SE, Hamman RF, Lachin JM, Walker EA, Nathan DM; Diabetes Prevention Program Research Group: Reduction in the incidence of type 2 diabetes with lifestyle intervention or metformin. N Engl J Med 2002;346:393-403.

6 Diabetes Prevention Program Research Group: Long-term effects of lifestyle intervention or metformin on diabetes development and microvascular complications over 15-year followup: the Diabetes Prevention Program Outcomes Study. Lancet Diabetes Endocrinol 2015;3: 866-875.

7 Look AHEAD Research Group; Wing RR, Bolin P, Brancati FL, et al: Cardiovascular effects of intensive lifestyle intervention in type 2 diabetes. N Engl J Med 2013;369:145-154.

8 Look AHEAD Research Group: Association of the magnitude of weight loss and changes in physical fitness with long-term cardiovascular disease outcomes in overweight or obese people with type 2 diabetes: a post-hoc analysis of the Look AHEAD randomised clinical trial. Lancet Diabetes Endocrinol 2016;4:913-921.

9 Estruch R, Ros E, Salas-Salvadó J, Covas MI, Corella D, Arós F, Gómez-Gracia E, Ruiz-Gutiérrez V, Fiol M, Lapetra J, Lamuela-Raventos RM, Serra-Majem L, Pintó X, Basora J, Muñoz MA, Sorlí JV, Martínez JA, Martínez-González MA; PREDIMED Study Investigators: Primary prevention of cardiovascular disease with a Mediterranean diet. N Engl J Med 2013;368: 1279-1290.
10 Salas-Salvadó J, Bulló M, Estruch R, Ros E, Covas MI, Ibarrola-Jurado N, Corella D, Arós F, Gómez-Gracia E, Ruiz-Gutiérrez V, Romaguera D, Lapetra J, Lamuela-Raventós RM, Serra-Majem L, Pintó X, Basora J, Muñoz MA, Sorlí JV, Martínez-González MA: Prevention of diabetes with Mediterranean diets: a subgroup analysis of a randomized trial. Ann Intern Med 2014;160:1-10.

11 Esposito K, Maiorino MI, Ciotola M, Di Palo C, Scognamiglio P, Gicchino M, Petrizzo M, Saccomanno F, Beneduce F, Ceriello A, Giugliano D: Effects of a Mediterranean-style diet on the need for antihyperglycemic drug therapy in patients with newly diagnosed type 2 diabetes: a randomized trial. Ann Intern Med 2009;151: 306-314.

12 Esposito K, Maiorino MI, Petrizzo M, Bellastella G, Giugliano D: The effects of a Mediterranean diet on the need for diabetes drugs and remission of newly diagnosed type 2 diabetes: follow-up of a randomized trial. Diabetes Care 2014;37:1824-1830.

13 Kempf K, Altpeter B, Berger J, Reuß O, Fuchs M, Schneider M, Gärtner B, Niedermeier K, Martin S: Efficacy of the Telemedical Lifestyle intervention Program TeLiPro in advanced stages of type 2 diabetes: a randomized controlled trial. Diabetes Care 2017;40:863-871. 